\title{
An Analysis of the Localized Airy-Laguerre-Gaussian Wave Packets Using Modified One-Parameter Point Symmetries ${ }^{+}$
}

\section{Jacob Matshwenyego Manale}

The Department of Mathematical Sciences, University of South Africa, Florida 1709, South Africa; manaljm@unisa.ac.za

† Presented at Symmetry 2017-The First International Conference on Symmetry, Barcelona, Spain, 16-18 October 2017.

Published: 12 January 2018

In their treatment of the Airy-Laguerre-Gaussian equation, Zhong, Belic and Zhang constructed an alternative solution for the azimuthal case; for the Airy case, they constructed finite pulses. The third case led to the Gaussian, considered to be un-integrable by some. To circumvent the need to construct solutions, which is susceptible to errors, as opposed to quadrature, we introduce the notion of modified one-parameter point symmetries. From this, we demonstrate that there is an error in the traditional solution for linear differential equations, such as the azimuthal equation, as Zhong, Belic and Zhang suspected, and provide the exact result. A finite solution for the Airy equation follows from the inverted monomials of its determining equation. Finally, we integrate the Gaussian integral. As an application, we propose how the analysis can be extended to Newtonian gravitation, and a technology for tapping space energy.

(C) 2018 by the author. Licensee MDPI, Basel, Switzerland. This article is an open access article distributed under the terms and conditions of the Creative Commons Attribution (CC BY) license (http://creativecommons.org/licenses/by/4.0/). 